\title{
A interdisciplinaridade como estratégia na prevenção da hipertensão arterial sistêmica em crianças: uma revisão sistemática
}

\author{
Interdisciplinarity as a strategy for the prevention of systemic \\ arterial hypertension in children: a systematic review
}

Luana Bernardi ${ }^{1}$

Mauricila de Campos França ${ }^{2}$

Audineia Martins Xavier ${ }^{2}$

Daiana Novello ${ }^{2}$

${ }^{1}$ Universidade Federal de Mato Grosso do Sul. Cidade

Universitária, Campus

Universitário. 79070-900

Campo Grande MS Brasil. luana_bernardi@

yahoo.com.br

${ }^{2}$ Universidade Estadual do

Centro Oeste. Guarapuava

PR Brasil.
Abstract Researches that developed interdisciplinary actions in schools seeking to modify the arteial pressure levels or salt and/or sodium consumption by children were analyzed. Studies available on the PubMed, ScienceDirect and Lilacs databases, associating the keywords: intervention study, nutrition intervention, schoolchildren, school health, blood pressure, sodium and salt were included. The research included articles from the last 15 years, in Portuguese, English and Spanish, which had preschool or school children as participants. With respect to the change of the blood pressure levels, 7 studies with positive and significant results and 6 without variation were identified. In terms of modification of the ingestion of food with high salt and/or sodium content, 4 studies revealed positive and significant results, and only one was unsuccessful. The conclusions drawn are that the interventions that use nutritional education combined with physical activities prevail in the literature. However, these actions proved to be more effective when implemented over a long period of time.

Key words Hypertension, Children, Sodium
Resumo Foram avaliadas, sistematicamente, pesquisas que desenvolveram ações interdisciplinares em escolas, visando modificar os níveis de pressão arterial ou o consumo de sal elou sódio por crianças. Foram incluídos estudos disponíveis nas bases de dados PubMed, ScienceDirect e Lilacs, associando-se as palavras-chave: intervention study, nutrition intervention, school children, school health, blood pressure, sodium e salt. A busca incluiu artigos em português, inglês e espanhol dos últimos 15 anos que tiveram como participantes crianças pré-escolares ou escolares. Com relação à mudança nos níveis de pressão arterial, foram identificados sete trabalhos com resultados positivos e significativos e seis que não apresentaram alteração. Quanto à modificação na ingestão de alimentos com alto teor de sal elou sódio, quatro trabalhos apresentaram resultados positivos e significativos e um sem sucesso. Concluiu-se que as intervenções que utilizam educação nutricional combinadas com atividade física predominam na literatura. Sendo que estas ações demonstraramse mais efetivas quando realizadas por um periodo longo de tempo.

Palavras-chave Hipertensão, Criança, Sódio 


\section{Introdução}

Atualmente, a hipertensão arterial sistêmica (HAS) é um problema de saúde pública ${ }^{1}$. A doença ocupa a $12^{\mathrm{a}}$ posição no ranking mundial de mortalidade $^{2}$ e, sua prevalência, no ano de 2013, foi estimada em $24,1 \%$ na população adulta brasileira que reside em capitais e no Distrito Federal do país ${ }^{3}$. Da mesma forma que em adultos, em crianças e adolescentes, a presença de elevações leves da pressão arterial ou mesmo de HAS são crescentes e muito mais comuns do que se verificava em anos anteriores ${ }^{4-8}$, fato que vem contribuindo para que esta doença deixe de ser exclusiva do adulto. A partir dos diversos fatores que podem influenciar a $\mathrm{HAS}^{9}$, tais como: perda de peso corporal; mudanças comportamentais nos hábitos alimentares; atividade física regular; moderação na ingestão de álcool; parar de fumar; controle do estresse e; ingestão reduzida de cloreto de sódio $(\mathrm{NaCl})$, cuidados interdisciplinares em saúde, incluindo aconselhamento dietético e de exercício físico, apoio social e a possibilidade de uma terapia comportamental cognitiva, passam a ser percebidos como estratégias fundamentais para o controle dessa doença ${ }^{10}$.

A moderação na ingestão de $\mathrm{NaCl}$ é visto como um dos principais benefícios para o tratamento da $\mathrm{HAS}^{11}$. Nesse sentido, sabendo-se do relevante impacto na saúde pública, muitas pesquisas vêm demonstrando a relação direta entre a ingestão de sódio e pressão arterial em crianças $^{6-8,12}$. Por acentuar o sabor dos alimentos $^{13}$, é comum um maior consumo de $\mathrm{NaCl}$ nas refeições familiares, fato que influencia negativamente o hábito alimentar infantil ${ }^{14}$. Além do contexto familiar, a escola também exerce papel relevante no consumo deste nutriente ${ }^{15}$. Visando colaborar para a correção desta prática, pesquisas vêm utilizando ações interdisciplinares nas escolas, incluindo aulas teóricas e didáticas e jogos de educação nutricional, como forma de reduzir o consumo excessivo de $\mathrm{NaCl}^{16-18}$, já que este local é reconhecido como altamente eficaz para o desenvolvimento de estratégias de intervenção em saúde.

As práticas interdisciplinares podem ser compreendidas como um esforço amplo, que excede as capacidades e recursos de um único profissional ou disciplina. Fundamentam-se em princípios de inclusão, com a colaboração de uma equipe de profissionais, devendo atender as necessidades complexas relacionadas à saúde das pessoas ${ }^{19}$. Entretanto, pouco se conhece so- bre a metodologia dos estudos e a eficácia de seus resultados na saúde do público infantil. Assim, faz-se necessária uma síntese dos estudos para possibilitar maior compreensão das diferentes estratégias disponíveis na literatura, o que pode subsidiar outras ações de prevenção e tratamento da HAS em crianças.

Neste contexto, o objetivo deste trabalho foi avaliar de forma sistemática as práticas interdisciplinares desenvolvidas em escolas, com o intuito de modificar os níveis de pressão arterial ou o consumo de sal e/ou sódio por crianças.

\section{Metodologia}

Foram avaliados artigos recentes (últimos 15 anos) da base de dados PubMed, ScienceDirect e Lilacs, publicados entre janeiro de 2000 e agosto de 2015. Os trabalhos deveriam propor pelo menos um dos seguintes objetivos: "modificações nos níveis de pressão arterial” ou "modificações na ingestão de alimentos com alto teor de sal e/ ou sódio" por escolares. Primeiramente, procedeu-se a busca de resumos, empregando o operador booleano and entre os seguintes descritores: intervention study, nutrition intervention, school children, school health, blood pressure, sodium e salt.

Os artigos foram selecionados pelo título e resumo, através dos seguintes critérios de inclusão: a) resultar em mudanças favoráveis/desfavoráveis nos níveis de pressão arterial ou na ingestão de sal e/ou sódio; b) ter amostra composta por crianças pré-escolares (2 a 6 anos), ou escolares (7 a 10 anos), ou, se tivesse grupos etários maiores, conter estratificações dentro dos citados padrões etários. Entretanto, se as amostras ou estratificações passavam dentro da faixa etária citada e excediam ligeiramente para menos ou para mais, também foram considerados; c) estar redigido em inglês, espanhol ou português; d) ter sido publicado entre 2000 e 2015 e; e) trabalhos realizados em ambiente escolar, por meio de práticas interdisciplinares. Foram adotados os seguintes critérios de exclusão: a) estudos de revisão; b) sem descrição metodológica completa (objetivos, métodos e resultados); c) estudos realizados com animais; d) ocorrer em ambiente fora da escola; e) não apresentar práticas interdisciplinares na metodologia e; f) não conter crianças na amostra. Também foi considerado critério de exclusão artigos duplicados, os quais foram eliminados manualmente. 


\section{Modificações nos níveis de pressão arterial ou na ingestão de sal e/ou sódio}

Para ambos os subtemas foram encontrados, respectivamente, um total de 718.215/519.132 artigos (PubMed: 76.206/65.927, ScienceDirect: $625.624 / 446.867$ e Lilacs: 16.385/6.338). Os descritores utilizados foram: intervention study, school health, school children e blood pressure para "modificações nos níveis de pressão arterial" e nutrition intervention, school children, school health, sodium e salt para "modificações na ingestão de sal e/ou sódio”. A exclusão dos trabalhos ocorreu da seguinte forma, respectivamente: estudo de revisão (85/78); metodologia incompleta (20/15); estudos com animais (6/2); ambientes fora da escola (150/214); não apresentar práticas interdisciplinares (350/250); não conter crianças na amostra (230/210); não ter sido publicado nos últimos quinze anos (717.318/518.320) e; artigos duplicados (12/4). Após a aplicação dos critérios de inclusão e exclusão, permaneceram, respectivamente: 44/39 artigos (PubMed: 35/30, ScienceDirect: 7/7 e Lilacs: 2/2), os quais foram lidos na íntegra. Em seguida, para o subtema

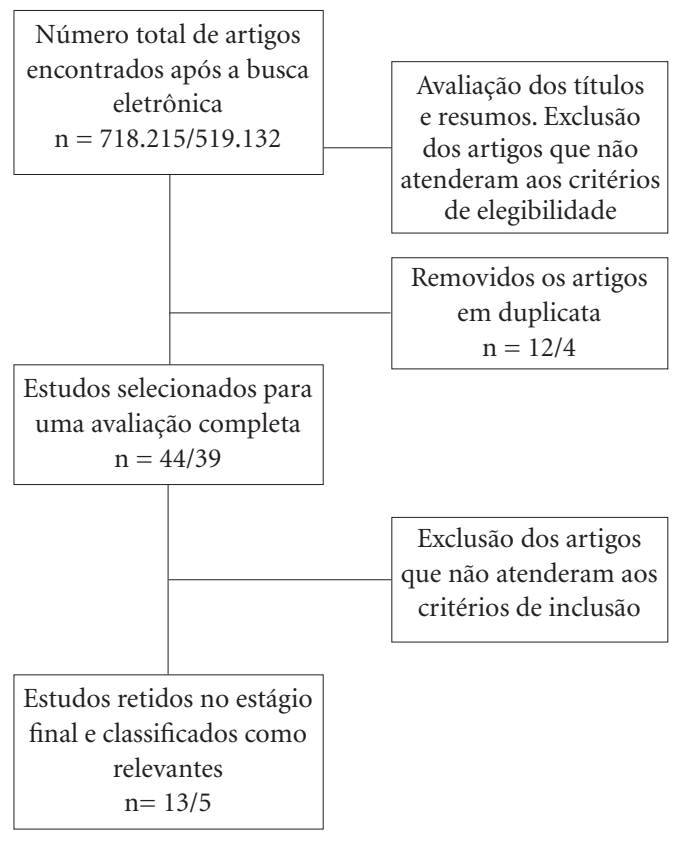

Figura 1. Fluxograma relacionado ao processo de seleção dos artigos com os temas "modificações nos níveis de pressão arterial” e "modificações na ingestão de sal e/ou sódio”, respectivamente. "modificações nos níveis de pressão arterial" foram excluídos mais 31 estudos que não estavam condizentes com o critério: apresentar resultados com mudanças favoráveis e/ou desfavoráveis nos níveis de pressão arterial. Já para o subtema "modificações na ingestão de sal e/ou sódio”, foram excluídos mais 34 estudos, os quais não estavam condizentes com o critério: apresentar mudanças favoráveis e/ou desfavoráveis na ingestão de sal e/ ou sódio. Dessa forma, permaneceram um total de 13/5 trabalhos (Figura 1), respectivamente, que foram tabulados, com a discriminação dos itens a seguir: autores, ano, amostra, país, objetivo do estudo, prática interdisciplinar e resultados (Quadros 1 e 2).

\section{Resultados}

\section{Modificações nos níveis de pressão arterial}

No Quadro 1 estão descritas as pesquisas que apresentavam como propostas de intervenção modificações nos níveis de pressão arterial de crianças. As práticas interdisciplinares descritas no Quadro 1 permitem verificar a abrangência das ações, além dos sujeitos nelas envolvidos. Dessa forma, quatro estudos envolveram pais/famílias dos escolares; um abrangeu os professores das escolas; quatro compreendiam pais e professores; um incluía pais, professores e comunidade local e; três envolveram pais, professores e outros funcionários da escola.

Foram abordados os seguintes temas relacionados às intervenções nutricionais: aplicação de atividade/programa de educação nutricional (13); sessões de aconselhamento nutricional individual e/ou em grupo (4); cursos de comportamento alimentar (3); inclusão de alimentos com melhor valor nutricional nas cantinas escolares (3); atividade complementar de jardinagem (2); elaboração de materiais para serem reproduzidos em sala de aula (7); desenvolvimento de receitas (2), grupos de discussão (3); uso de diários alimentares ilustrativos (1); transmissão de informações através de histórias infantis (1) e; elaboração de materiais educativos (imãs de geladeira, cartões plastificados) (4).

As ações de atividade física compreenderam os seguintes assuntos: fortalecimento muscular (2); alongamento (2); atividades aeróbias (6); jogos com bola (3); uso de bicicleta (1); esportes (4); dança (4); aulas de natação (1); brincadeiras ao ar livre (1) e; orientações para redução do tempo gasto com televisão e jogos de computador (3). 
Quadro 1. Artigos que apresentaram a proposta de modificações nos níveis de pressão arterial de crianças.

\begin{tabular}{|c|c|c|c|c|}
\hline Autores (ano) & Amostra/País & Objetivo(s) & Prática interdisciplinar & Resultados \\
\hline $\begin{array}{l}\text { Angelopoulo et } \\
\text { al. }(2009)^{20}\end{array}$ & $\begin{array}{l}\mathrm{n}=646, \mathrm{M} \\
=286, \mathrm{~F}= \\
360, \text { Média de } \\
\text { idade: } 10,25 \\
\pm 0,44 \text { anos, } \\
\text { Grécia }\end{array}$ & \begin{tabular}{|l} 
Analisar um \\
programa de \\
intervenção, com \\
base na Teoria do \\
Comportamento \\
Planejado (TCP), em \\
índices de OI e PA. \\
\end{tabular} & $\begin{array}{l}\text { Utilizavam-se livros (alunos) e } \\
\text { manuais (professores), abordando } \\
\text { atividades de imagem corporal, } \\
\text { autoestima, nutrição, AF aptidão e } \\
\text { questões ambientais. } \\
\text { Tempo de intervenção: } 12 \text { meses. } \\
\text { Público atendido: crianças, pais e } \\
\text { professores. }\end{array}$ & $\begin{array}{l}\text { GI: diminuição } \\
\text { da PAS }(\mathrm{p}= \\
0,02) \text { e PAD } \\
(\mathrm{p}=0,01) .\end{array}$ \\
\hline $\begin{array}{l}\text { Reinehr et al. } \\
(2010)^{21}\end{array}$ & $\begin{array}{l}\mathrm{n}=66, \mathrm{M}= \\
28, \mathrm{~F}=38 \\
\text { Média de } \\
\text { idade: } 11,5 \pm \\
1,6 \text { anos, } \\
\text { Alemanha }\end{array}$ & $\begin{array}{l}\text { Demonstrar a } \\
\text { efetividade de uma } \\
\text { intervenção no estilo } \\
\text { de vida de crianças } \\
\text { com sobrepeso. }\end{array}$ & $\begin{array}{l}\text { Realizou-se a "Obeldicks light" } \\
\text { com uma equipe interdisciplinar. } \\
\text { O treinamento focava em AF, EN } \\
\text { e AC, com sessões individuais e } \\
\text { em grupos para as crianças e suas } \\
\text { famílias. } \\
\text { Tempo de intervenção: } 6 \text { meses. } \\
\text { Público atendido: crianças e pais. }\end{array}$ & $\begin{array}{l}\text { GI: diminuição } \\
\text { da PAS }(\mathrm{p}< \\
0,00) \text { e PAD } \\
(\mathrm{p}<0,00) .\end{array}$ \\
\hline $\begin{array}{l}\text { Hollar et al. } \\
(2010)^{22}\end{array}$ & $\begin{array}{l}\mathrm{n}=3.769, \mathrm{M} \\
=1.847, \mathrm{~F}= \\
1.922 \\
4 \text { a } 13 \text { anos, } \\
\text { EUA }\end{array}$ & $\begin{array}{l}\text { Reduzir taxas de OI, } \\
\text { melhorar a saúde } \\
\text { e desempenho } \\
\text { acadêmico } \\
\text { (estratégias multi- } \\
\text { níveis). Modificar } \\
\text { o conhecimento e } \\
\text { comportamentos } \\
\text { associados à OI, } \\
\text { além de contribuir } \\
\text { para uma } \\
\text { alimentação e vida. } \\
\text { saudável. } \\
\end{array}$ & $\begin{array}{l}\text { Inclusão de ingredientes nutritivos } \\
\text { na escola, incentivando a nutrição, } \\
\text { estilo de vida saudável, aumento da } \\
\text { AF e promoção de outras atividades, } \\
\text { como a agroecologia. } \\
\text { Tempo de intervenção: } 2 \text { anos. } \\
\text { Público atendido: crianças, pais, } \\
\text { professores e funcionários das } \\
\text { escolas. }\end{array}$ & $\begin{array}{l}\text { F: aumento } \\
\text { da PAS no GC } \\
(p<0,00) \\
\text { M: aumento na } \\
\text { PAS do GI e GC } \\
(p<0,00) . \\
\text { F e M: aumento } \\
\text { na PAD no GI e } \\
\text { GC }(p<0,00) .\end{array}$ \\
\hline $\begin{array}{l}\text { Hollar et al. } \\
(2010)^{23}\end{array}$ & $\begin{array}{l}\mathrm{n}=2.494, \mathrm{M} \\
=1.222, \mathrm{~F}= \\
1.272 \\
6 \text { a } 13 \text { anos, } \\
\text { EUA }\end{array}$ & $\begin{array}{l}\text { Melhorar o estado } \\
\text { geral de saúde (IMC } \\
\text { e PA) e desempenho } \\
\text { acadêmico, por } \\
\text { meio de estratégias } \\
\text { replicadoras e } \\
\text { integradas nas } \\
\text { escolas públicas. }\end{array}$ & $\begin{array}{l}\text { Uso de ingredientes nutritivos e } \\
\text { integrais nas refeições escolares; } \\
\text { programa sobre EN e estilo de vida } \\
\text { saudável; aumento dos níveis de AF } \\
\text { e jardinagem. } \\
\text { Tempo de intervenção: } 2 \text { anos. } \\
\text { Público atendido: crianças, pais, } \\
\text { professores e funcionários das } \\
\text { escolas. }\end{array}$ & $\begin{array}{l}\text { F: redução } \\
\text { da PAS no GI } \\
\text { ( } p=0,03) ; \mathrm{F}: \\
\text { redução na PAD } \\
\text { no GI ( } \mathrm{p} \leq 0,05) \text {. } \\
\text { M: alterações } \\
\text { não } \\
\text { significativas na } \\
\text { PAD no GI. }\end{array}$ \\
\hline $\begin{array}{l}\text { Plachta- } \\
\text { Danielzik et al. } \\
(2011)^{24}\end{array}$ & $\begin{array}{l}\mathrm{n}=1.192, \mathrm{M} \\
=596 \\
\mathrm{~F}=596,6 \\
\text { a } 14 \text { anos, } \\
\text { Alemanha }\end{array}$ & $\begin{array}{l}\text { Intervir no peso, } \\
\text { estilo de vida e } \\
\text { PA como parte do } \\
\text { Estudo de Prevenção } \\
\text { da Obesidade Kiel } \\
\text { (KOPS). }\end{array}$ & $\begin{array}{l}\text { Orientações de alimentação } \\
\text { saudável, AF e redução de TV, com } \\
\text { auxílio de histórias infantis, jogos } \\
\text { interativos e preparo de café da } \\
\text { manhã saudável. Jogos de corrida } \\
\text { eram oferecidos no pátio da escola. } \\
\text { Tempo de intervenção: } 8 \text { anos. } \\
\text { Público atendido: crianças, } \\
\text { adolescentes, pais e professores. }\end{array}$ & $\begin{array}{l}\text { Pós- } \\
\text { intervenção: } \\
\text { maior } \\
\text { prevalência de } \\
\text { PA elevada no } \\
\text { GC }(13.3 \%) \\
\text { do que no GI } \\
(10.8 \%) \\
(p>0,05) . \\
\end{array}$ \\
\hline $\begin{array}{l}\text { Choudhry et } \\
\text { al. }(2011)^{25}\end{array}$ & $\begin{array}{l}\mathrm{n}=40, \mathrm{M}= \\
16, \mathrm{~F}=24,5 \mathrm{a} \\
12 \text { anos, EUA }\end{array}$ & $\begin{array}{l}\text { Estudar a } \\
\text { intervenção } \\
\text { Power-Up, para } \\
\text { diminuir o risco de } \\
\text { OI entre crianças } \\
\text { afro-americanas, } \\
\text { com os princípios } \\
\text { da pesquisa } \\
\text { participativa baseada } \\
\text { na comunidade. } \\
\end{array}$ & $\begin{array}{l}\text { Sessões semanais que versavam } \\
\text { sobre EN e AF. Eram lideradas por } \\
\text { professores das escolas com suporte } \\
\text { dos pesquisadores. } \\
\text { Tempo de intervenção: } 14 \text { semanas } \\
\text { Público atendido: crianças e seus } \\
\text { pais. }\end{array}$ & $\begin{array}{l}\text { Pós- } \\
\text { intervenção: } \\
\text { diminuição } \\
\text { da prevalência } \\
\text { de PAS e PAD } \\
\text { elevada } \\
(\mathrm{p}>0,05) .\end{array}$ \\
\hline
\end{tabular}


Quadro 1. continuação

\begin{tabular}{|c|c|c|c|c|}
\hline Autores (ano) & Amostra/País & Objetivo(s) & Prática interdisciplinar & Resultados \\
\hline $\begin{array}{l}\text { Schaefer et al. } \\
(2011)^{26}\end{array}$ & $\begin{array}{l}\mathrm{n}=76, \mathrm{M}= \\
25, \mathrm{~F}=51 \\
\text { Média de } \\
\text { idade: } \\
11,8 \pm \\
1,8 \text { anos, } \\
\text { Alemanha }\end{array}$ & $\begin{array}{l}\text { Investigar o } \\
\text { benefício de } \\
\text { uma intervenção } \\
\text { interdisciplinar no } \\
\text { estilo de vida de } \\
\text { crianças com excesso } \\
\text { de peso. }\end{array}$ & $\begin{array}{l}\text { Aplicava-se a "Obeldicks light" } \\
\text { baseada na AF, EN e AC, incluindo } \\
\text { atendimento psicológico para } \\
\text { a criança e família. Ações para } \\
\text { redução no tempo de TV/ } \\
\text { computador, além de um curso } \\
\text { de comportamento alimentar e } \\
\text { nutricional. } \\
\text { Tempo de intervenção: } 6 \text { meses. } \\
\text { Público atendido: crianças com } \\
\text { excesso de peso e seus pais. }\end{array}$ & $\begin{array}{l}\text { Redução na PAS } \\
(\mathrm{p}<0,00) \text { e } \\
\operatorname{PAD}(\mathrm{p}<0,00) .\end{array}$ \\
\hline $\begin{array}{l}\text { Rush et al. } \\
(2012)^{27}\end{array}$ & $\begin{array}{l}\mathrm{n}=1.352, \\
\mathrm{M}=686, \mathrm{~F} \\
=666,5 \text { a } 12 \\
\text { anos, Nova } \\
\text { Zelândia }\end{array}$ & $\begin{array}{l}\text { Avaliar o projeto } \\
\text { "Energize", que tem } \\
\text { objetivos de: analisar } \\
\text { a PA e composição } \\
\text { corporal; identificar } \\
\text { preditores do } \\
\text { aumento da gordura } \\
\text { corporal e PA em } \\
\text { relação à idade, sexo, } \\
\text { etnia, ruralidade e } \\
\text { privação social. }\end{array}$ & $\begin{array}{l}\text { Eram utilizadas brincadeiras e jogos } \\
\text { de AF, iniciativas para alimentação } \\
\text { saudável e modificação de alimentos } \\
\text { vendidos nas cantinas escolares. } \\
\text { Tempo de intervenção: } 2 \text { anos. } \\
\text { Público atendido: escolares, } \\
\text { professores, pais e comunidade } \\
\text { local. }\end{array}$ & $\begin{array}{l}\text { Não houve } \\
\text { diferença } \\
\text { significativa no } \\
\text { GI e GC entre } \\
\text { as crianças de } 5 \\
\text { a } 7 \text { anos na PAS } \\
(\mathrm{p}=0,79) \text { e PAD } \\
(\mathrm{p}=0,68) \text { e entre } \\
\text { as crianças de } \\
10 \text { a } 12 \text { anos na } \\
\text { PAS }(\mathrm{p}=0,16) \text { e } \\
\text { PAD }(\mathrm{p}=0,22) .\end{array}$ \\
\hline $\begin{array}{l}\text { Vásquez et al. } \\
(2013)^{28}\end{array}$ & $\begin{array}{l}\mathrm{n}=120, \mathrm{M}= \\
60, \mathrm{~F}=60,8 \mathrm{a} \\
13 \text { anos, Chile }\end{array}$ & $\begin{array}{l}\text { Mensurar o impacto } \\
\text { de uma intervenção } \\
\text { interdisciplinar } \\
\text { no sistema escolar, } \\
\text { incluindo o exercício } \\
\text { de força muscular, } \\
\text { EN e AC para } \\
\text { crianças obesas. }\end{array}$ & $\begin{array}{l}\text { Sessões de EN, exercícios de } \\
\text { treinamento de força e apoio } \\
\text { psicológico para } 2 \text { grupos de estudo. } \\
\text { Tempo de intervenção: } 6 \text { meses. } \\
\text { Público atendido: crianças e seus } \\
\text { pais. }\end{array}$ & $\begin{array}{l}\text { Nos } 3 \text { primeiros } \\
\text { meses: aumento } \\
\text { da PA }(\mathrm{p}=0,02) \\
\text { no grupo } 2 . \\
\text { Entre } 3 \text { e } 6 \\
\text { meses: aumento } \\
\text { na PA ( } \mathrm{p}=0,003 \text { ) } \\
\text { no grupo } 1 \text { e } \\
\text { redução na PA } \\
\text { (p=0,01) no } \\
\text { grupo } 2 \text {, que } \\
\text { recebeu AF. }\end{array}$ \\
\hline $\begin{array}{l}\text { Militão et al. } \\
(2013)^{29}\end{array}$ & $\begin{array}{l}\mathrm{n}=34, \mathrm{M}= \\
16, \mathrm{~F}=18,9 \mathrm{a} \\
11 \text { anos, Brasil }\end{array}$ & $\begin{array}{l}\text { Avaliar os efeitos de } \\
\text { um programa de } \\
\text { AF de lazer, junto } \\
\text { com um diário } \\
\text { ilustrativo, no perfil } \\
\text { cardiovascular } \\
\text { de escolares } \\
\text { com sobrepeso e } \\
\text { obesidade. }\end{array}$ & $\begin{array}{l}\text { O GI realizava atividades } \\
\text { recreativas, orientações sobre } \\
\text { hábitos saudáveis e elaboravam um } \\
\text { diário ilustrativo de alimentação } \\
\text { saudável, além de reduzir } \\
\text { comportamentos sedentários. } \\
\text { Tempo de intervenção: } 10 \text { semanas. } \\
\text { Público atendido: crianças e } \\
\text { professores. }\end{array}$ & $\begin{array}{l}\text { GI: redução na } \\
\text { PAS }(p<0,01) \\
\text { PA entre GI e } \\
\text { GC }(p>0,05)\end{array}$ \\
\hline $\begin{array}{l}\text { Muros et al. } \\
(2013)^{30}\end{array}$ & $\begin{array}{l}\mathrm{n}=54, \mathrm{M}= \\
25, \mathrm{~F}=29,10 \\
\text { a } 11 \text { anos, } \\
\text { Espanha }\end{array}$ & $\begin{array}{l}\text { Determinar o efeito } \\
\text { da EN combinada } \\
\text { com sessões de AF } \\
\text { extracurricular na } \\
\text { melhora ds saúde } \\
\text { (educação primária). }\end{array}$ & $\begin{array}{l}\text { Sessões de AF extracurricular } \\
\text { realizada } 2 \text { vezes/semana e EN } \\
\text { para os estudantes e seus pais, } \\
\text { informando-os sobre um estilo de } \\
\text { vida e dietas saudáveis. } \\
\text { Tempo de intervenção: } 6 \text { meses. } \\
\text { Público atendido: crianças e seus } \\
\text { pais. }\end{array}$ & $\begin{array}{l}\text { GI: redução na } \\
\text { PAS e PAD } \\
(\mathrm{p}<0,05)\end{array}$ \\
\hline
\end{tabular}


Quadro 1. continuação

\begin{tabular}{|c|c|c|c|c|}
\hline Autores (ano) & Amostra/País & Objetivo(s) & Prática interdisciplinar & Resultados \\
\hline $\begin{array}{l}\text { Hrafnkelsson } \\
\text { et al. }(2014)^{31}\end{array}$ & $\begin{array}{l}\mathrm{n}=268, \mathrm{M}= \\
134, \mathrm{~F}=134, \\
7 \text { a } 9 \text { anos, } \\
\text { Islândia }\end{array}$ & $\begin{array}{l}\text { Relatar o efeito de } \\
\text { uma intervenção } \\
\text { de dois anos no } \\
\text { ambiente escolar, por } \\
\text { meio da integração } \\
\text { de AF e EM sobre } \\
\text { fatores de risco para } \\
\text { DCV }\end{array}$ & $\begin{array}{l}\text { Programa de AF, aumentando } \\
\text { progressivamente o tempo de } \\
\text { prática de atividades, e EN aos } \\
\text { estudantes, pais, professores e } \\
\text { pessoal do serviço de alimentação. } \\
\text { Tempo de intervenção: } 2 \text { anos. } \\
\text { Público atendido: escolares, } \\
\text { professores, pais e pessoal do } \\
\text { serviço de alimentação escolar. }\end{array}$ & $\begin{array}{l}\text { GI e GC: } \\
\text { aumento na PAS } \\
\text { e PAD. Aumento } \\
\text { maior no GC, } \\
\text { na PAS (p > } \\
0,05) \text { e PAD } \\
(p>0,05) .\end{array}$ \\
\hline $\begin{array}{l}\text { He et al. } \\
(2015)^{32}\end{array}$ & $\begin{array}{l}\mathrm{n}=279, \mathrm{M} \\
=134, \mathrm{~F}= \\
145, \text { Média } \\
\text { de idade: } 10,1 \\
\pm 0,5 \text { anos, } \\
\text { China }\end{array}$ & $\begin{array}{l}\text { Determinar se } \\
\text { um programa de } \\
\text { educação dirigido } \\
\text { a escolares poderia } \\
\text { reduzir o consumo } \\
\text { de sal nestes e em } \\
\text { suas famílias. }\end{array}$ & $\begin{array}{l}\text { Aulas de educação em saúde, com } \\
\text { foco na redução do consumo de } \\
\text { sal, incluindo palestras, reuniões de } \\
\text { classe, jogos, competições de escrita } \\
\text { e desenhos, lições de casa e entrega } \\
\text { de materiais informativos. } \\
\text { Tempo de intervenção: } 3,5 \text { meses. } \\
\text { Público atendido: crianças, pais, } \\
\text { avós e professores. }\end{array}$ & $\begin{array}{l}\text { GI e GC: } \\
\text { aumento na } \\
\text { PAS e PAD. } \\
\text { PAS: menor } \\
\text { aumento no GI } \\
\text { comparado ao } \\
\text { GC }(p<0,05) \text {. }\end{array}$ \\
\hline
\end{tabular}

${ }^{*}$ M: Sexo masculino, F: Sexo feminino, n: tamanho amostral, EUA: Estados Unidos da América, EN: educação nutricional, PA: pressão arterial, PAS: pressão arterial sistólica, PAD: pressão arterial diastólica, OI: obesidade infantil, AF: atividade física, AC: aconselhamento comportamental, GC: grupo controle, GI: grupo intervenção, NI: não intervenção, IMC: índice de massa corporal, USDA: Departamento de Agricultura dos Estados Unidos, TV: televisão, DCV: doença cardiovascular.

Quanto às intervenções destinadas aos outros indivíduos do ambiente escolar, destacam-se: palestras/encontros na escola com a família (11); sessões de aconselhamento nutricional individual e psicológico com os pais (2); aconselhamento e estímulo aos pais para incentivar seus filhos à prática de atividade física (2) e; divulgação e incentivo aos pais para adoção de hábitos alimentares adequados e prática de exercícios (7). Além disso, em sete estudos os professores das escolas tiveram reuniões e palestras sobre os benefícios da substituição de alimentos com alto teor em açúcar e gordura por outros mais nutritivos em cantinas, com o intuito de aderir às condutas alimentares saudáveis. Também, foi abordada a importância dos professores realizarem o café da manhã, treinamentos e sessões de apoio para auxiliar na aplicação de materiais em sala de aula, desenvolvimento de atividades práticas e elaboração de materiais educativos. Em três estudos ${ }^{20,27,32}$ houve a participação direta dos professores nas intervenções de educação nutricional e, em apenas uma das pesquisas ${ }^{26}$ o professor de educação física teve a responsabilidade de planejar as atividades recreacionais. Nestes quatro trabalhos, os educadores receberam treinamento prévio da equipe de pesquisadores, visando a aplicação das ações em sala de aula. Já em outros cinco traba$\operatorname{lhos}^{22,23,24,25,31}$, os professores apenas auxiliaram a execução das atividades com as crianças, a partir de explicações prévias dos profissionais.

Destaca-se a participação de um grande número de profissionais na aplicação das intervenções, estando presentes dietistas ${ }^{22,23,25,27}$, nutricionistas $^{24,25,27}$, professores de educação física ${ }^{29,27,31}$, psicólogos $^{25,28}$, endocrinologistas ${ }^{25}$, especialistas em educação e ciência do esporte ${ }^{30}$ e educadores em saúde ${ }^{32}$. No que condiz ao envolvimento de equipes interdisciplinares, dois estudos ${ }^{21,26}$ citaram envolvê-las nas pesquisas, abrangendo pediatras, assistentes dietéticos, psicólogos e fisiologistas do exercício.

Com relação à redução nos níveis de pressão arterial, a partir de estratégias interdisciplinares de intervenção, foram identificados sete trabalhos que apresentaram resultado positivo e significativo na correlação entre a intervenção e esse desfecho. Porém, seis pesquisas não registraram redução nos níveis de pressão arterial com o uso de estratégias multidisciplinares.

\section{Modificações na ingestão de sal e/ou sódio}

No Quadro 2 estão descritas as pesquisas que apresentavam como propostas de intervenção modificações no consumo alimentar de sódio e/ ou sal de crianças. 
Quadro 2. Artigos que apresentaram a proposta de modificações no consumo alimentar de sódio e/ou sal de crianças.

\begin{tabular}{|c|c|c|c|c|}
\hline $\begin{array}{l}\text { Autores } \\
\text { (ano) }\end{array}$ & Amostra/País & Objetivo & Prática interdisciplinar & Resultados \\
\hline $\begin{array}{l}\text { Colín- } \\
\text { Ramírez et } \\
\text { al. }(2009)^{33}\end{array}$ & $\begin{array}{l}\mathrm{n}=498, \mathrm{M}= \\
267 \\
\mathrm{~F}=231,8 \text { a } 10 \\
\text { anos, } \\
\text { México }\end{array}$ & $\begin{array}{l}\text { Avaliar ações de } \\
\text { prevenção de doença } \\
\text { cardiovascular em } \\
\text { crianças (idade } \\
\text { escolar). }\end{array}$ & $\begin{array}{l}\text { Atividades para melhorar os hábitos } \\
\text { alimentares e AF. Capacitação de } \\
\text { professores de sala de aula e de } \\
\text { educação física para aplicação às } \\
\text { crianças. } \\
\text { Tempo de intervenção: } 12 \text { meses. } \\
\text { Público atendido: crianças, pais e } \\
\text { professores. }\end{array}$ & $\begin{array}{l}\text { GI: redução do } \\
\text { consumo de sódio } \\
(p<0,00) . \\
\text { GC: aumento do } \\
\text { consumo de sódio } \\
(p<0,00) .\end{array}$ \\
\hline $\begin{array}{l}\text { Bacardí- } \\
\text { Gascon et } \\
\text { al. }(2012)^{34}\end{array}$ & $\begin{array}{l}\mathrm{n}=532, \mathrm{M} \\
=272, \mathrm{~F}= \\
260, \text { Média } \\
\text { de idade: } 8,5 \\
\pm 0,73 \text { anos } \\
\text { México }\end{array}$ & $\begin{array}{l}\text { Estudar o efeito } \\
\text { de intervenção } \\
\text { (6 meses) e } \\
\text { acompanhamento } \\
\text { (18 meses) sobre } \\
\text { o IMC, consumo } \\
\text { alimentar e AF de } \\
\text { crianças (ensino } \\
\text { fundamental). }\end{array}$ & $\begin{array}{l}\text { Sessões interativas de EN e AF para } \\
\text { um consumo alimentar saudável, } \\
\text { redução do tempo de TV e aumento } \\
\text { na participação em esportes e jogos. } \\
\text { Tempo de intervenção: } 2 \text { anos. } \\
\text { Público atendido: crianças, pais, } \\
\text { professores e funcionários. }\end{array}$ & $\begin{array}{l}\text { Redução do } \\
\text { consumo de } \\
\text { lanches contendo } \\
\text { alta quantidade } \\
\text { de sal nas cantinas } \\
\text { escolares ( } \mathrm{p}=0,03 \text { ) } \\
\text { e ambiente } \\
\text { familiar }(\mathrm{p}=0,01) \text {. }\end{array}$ \\
\hline $\begin{array}{l}\text { Cotter et al. } \\
(2013)^{18}\end{array}$ & $\begin{array}{l}\mathrm{n}=139, \mathrm{M}= \\
63, \mathrm{~F}=76,10 \\
\text { a } 12 \text { anos, } \\
\text { Portugal }\end{array}$ & $\begin{array}{l}\text { Avaliar a excreção } \\
\text { urinária de sódio ( } 24 \\
\text { horas) em escolares. } \\
\text { Analisar a influência } \\
\text { do sal na PA após } \\
\text { três intervenções } \\
\text { educacionais. }\end{array}$ & $\begin{array}{l}\text { As intervenções consistiram em } \\
\text { nenhuma ação adicional (GC); aulas } \\
\text { semanais sobre alta ingestão de sal } \\
\text { (GT) e, aulas teóricas sobre este tema } \\
\text { com práticas de plantio e coleta de } \\
\text { ervas para substituição do sal em casa } \\
\text { (GP). } \\
\text { Tempo de intervenção: } 6 \text { meses. } \\
\text { Público atendido: crianças e } \\
\text { professores. }\end{array}$ & $\begin{array}{l}\text { GP: ingestão de } \\
\text { sódio reduzida } \\
(\mathrm{p}<0,00) \\
\text { comparado ao GT } \\
\text { e GC }(p>0,05) .\end{array}$ \\
\hline $\begin{array}{l}\text { Morgan et } \\
\text { al. }(2014)^{35}\end{array}$ & $\begin{array}{l}\mathrm{n}=105, \mathrm{M}= \\
55, \mathrm{~F}=50,9 \mathrm{a} \\
11 \text { anos, EUA }\end{array}$ & $\begin{array}{l}\text { Melhorar os hábitos } \\
\text { alimentares, a } \\
\text { imagem corporal, } \\
\text { autoestima e } \\
\text { motivação das } \\
\text { crianças através } \\
\text { do uso de avatares, } \\
\text { além de aumentar a } \\
\text { prática de AF. } \\
\end{array}$ & $\begin{array}{l}\text { O programa incluiu o incentivo } \\
\text { às boas práticas alimentares e AF. } \\
\text { Utilizaram-se avatares como uma } \\
\text { ferramenta de compreensão e } \\
\text { desenvolvimento de corpos saudáveis } \\
\text { para crianças próximas à puberdade. } \\
\text { Tempo de intervenção: } 10 \text { semanas. } \\
\text { Público atendido: crianças e } \\
\text { professores. }\end{array}$ & $\begin{array}{l}\text { GI e GC: sem } \\
\text { alteração no } \\
\text { consumo de } \\
\text { alimentos com } \\
\text { alto teor de sódio } \\
(\mathrm{p}=0,60) .\end{array}$ \\
\hline $\begin{array}{l}\text { He et al. } \\
(2015)^{32}\end{array}$ & $\begin{array}{l}\mathrm{n}=279, \mathrm{M} \\
=134, \mathrm{~F}= \\
145, \text { Média } \\
\text { de idade: } 10,1 \\
\pm 0,5 \text { anos, } \\
\text { China }\end{array}$ & $\begin{array}{l}\text { Determinar se } \\
\text { um programa de } \\
\text { educação dirigido } \\
\text { a escolares poderia } \\
\text { reduzir o consumo } \\
\text { de sal nestes e em } \\
\text { suas famílias. }\end{array}$ & $\begin{array}{l}\text { Aulas de educação em saúde, com } \\
\text { foco na redução do consumo de } \\
\text { sal, incluindo palestras, reuniões de } \\
\text { classe, jogos, competições de escrita e } \\
\text { desenhos, lições de casa e entrega de } \\
\text { materiais informativos. } \\
\text { Tempo de intervenção: } 3,5 \text { meses. } \\
\text { Público atendido: crianças, pais, avós } \\
\text { e professores. }\end{array}$ & $\begin{array}{l}\text { GI: ingestão de } \\
\text { sódio reduzida } \\
(\mathrm{p}<0,001) \\
\text { comparado ao GC. }\end{array}$ \\
\hline
\end{tabular}

*M: Sexo masculino, F: Sexo feminino, n: tamanho amostral, EN: educação nutricional, EUA: Estados Unidos da América, PA: pressão arterial, AF: atividade física, GC: grupo controle, GI: grupo intervenção, GT: Grupo teórico, GP: Grupo prático, IMC: índice de massa corporal, mg: miligrama, g: grama.

Em todos os estudos avaliados foi possível verificar que as intervenções foram direcionadas a outros indivíduos, além das crianças. Assim, as seguintes intervenções foram verificadas com outros sujeitos: palestras/encontros no ambiente escolar com os pais (2), sessões de educação nutricional para ampliar o conhecimento e incentivar a adoção de um estilo de vida saudável com 
a família (2), tarefas para os pais realizarem em casa (1), sugestões de cardápios, incluindo lanches saudáveis, para a cantina das escolas (1) e, entrega de materiais informativos aos pais e avós (1). Além disso, os professores, que também foram envolvidos nas intervenções, passaram por reuniões e palestras para explicar sobre os objetivos e importância da implantação dos programas de intervenção na escola (1), capacitação e recebimento de um manual de procedimentos para implementação das atividades de intervenção em sala de aula (2), encontros com professores e o conselho escolar para discutir questões de melhoria no ambiente escolar e incentivar um estilo de vida saudável (1).

As intervenções direcionadas à população de escolares, com relação às atividades de educação nutricional abrangeram: atividade/programa de educação nutricional (5); aulas interativas para aumentar o consumo de alimentos saudáveis (4); sessões sobre o valor nutricional dos alimentos (1); inclusão de alimentos com maior valor nutricional (1), tanto no almoço quanto nos lanches servidos nas cantinas das escolas; atividade complementar de jardinagem sobre o plantio e coletiva de ervas (1); aulas semanais teóricas (2); palestras pró-ativas e interativas (1); exercícios em sala de aula (1); competições de escrita e desenhos (1); uso de representações específicas para cada gênero, criadas em computador, por meio de avatares (as representações eram criadas pelas crianças, que alteravam a aparência física do avatar de acordo com a sua percepção de corpo saudável) (1) e; brincadeiras lúdicas (ex. semáforo dos alimentos) (2). Já as ações de atividade física compreenderam: discussão com funcionários da escola para melhorar as instalações para atividade física na escola e oferecê-la antes e depois do horário escolar (1); aumentar o número de alunos inscritos em atividades de jogos e esportes (1); oferta de atividade física em sala de aula por meio do uso de DVD (ex. "6 Fit Kids") (1); sessões sobre os benefícios do exercício (2); aulas de educação física com diferentes intensidades (moderada à vigorosa) (2) e; orientações para redução do tempo gasto com televisão (1).

Quanto aos profissionais envolvidos na aplicação das intervenções, verificou-se a presença de instrutor de atividade física ${ }^{37}$, nutricionista ${ }^{35}$, professor de educação física ${ }^{33,34,35}$ e educadores em saúde ${ }^{32}$. No que condiz ao envolvimento de equipes interdisciplinares, somente um dos estu$\operatorname{dos}^{33}$ citou abrangê-las nas pesquisas, compreendendo médico, odontólogo, psicólogo, enfermeira e assistente social. Em dois dos trabalhos ${ }^{32,35}$ foi constatada a participação dos professores na realização das ações, sendo que recebiam treinamento prévio pela equipe do programa, para que as ações pudessem ocorrer em sala de aula. Já em outros três estudos ${ }^{18,33,34}$, os professores apenas auxiliaram a execução das atividades com as crianças, a partir de explicações prévias e presença dos profissionais durante as atividades.

Quanto à mudança na ingestão de alimentos com elevado conteúdo de sal e/ou sódio, a partir de estratégias interdisciplinares de intervenção, foram identificados quatro pesquisas que apresentaram resultado positivo e significativo na correlação entre a intervenção e esse desfecho. Já, quanto aos trabalhos que não obtiveram sucesso nesta mudança com o uso de estratégias multidisciplinares foram identificados apenas um.

\section{Relação entre a prática interdisciplinar e a efetividade dos resultados}

No Quadro 3 estão apresentadas as relações entre o tipo de prática interdisciplinar de cada estudo avaliado e seu efeito sobre a mudança no comportamento alimentar, com foco na redução no consumo de alimentos com alto teor de sal e/ ou sódio e, principalmente, seu impacto sobre alterações na pressão arterial de crianças.

Quanto ao efeito na pressão arterial, todos os estudos analisados desenvolveram ações interdisciplinares com base na associação de atividades de educação nutricional e atividade física. Do total, quatro relacionaram educação nutricional, atividade física e sessões educativas em sala de aula, dois associaram educação nutricional, atividade física e ações de jardinagem, três combinaram educação nutricional e atividade física, apenas um trabalho associou educação nutricional com sessões educativas em sala de aula e, três trabalhos aderiram à educação nutricional, atividade física e apoio psicológico, sendo este último o programa que obteve os resultados mais efetivos.

Com base na análise metodológica dos estudos que buscaram exercer efeito sobre o comportamento alimentar, dois associaram atividades de educação nutricional, atividade física e sessões educativas em sala de aula, dois combinaram educação nutricional com sessões educativas em sala de aula e, um integrou educação nutricional e atividade física, não possibilitando determinar qual programa alcançou maior efetividade em seus resultados, uma vez que somente um trabalho não conseguiu alterar o hábito dietético relacionado ao consumo de alimentos com alto teor em sódio. 
Quadro 3. Efetividade das práticas interdisciplinares sobre o comportamento alimentar e redução da pressão arterial das crianças avaliadas nos artigos.

\begin{tabular}{|c|c|c|c|c|}
\hline \multirow{2}{*}{ Prática interdisciplinar } & \multicolumn{2}{|c|}{ Efeito no comportamento alimentar } & \multicolumn{2}{|c|}{ Efeito na PA } \\
\hline & Efetivo & Não efetivo & Efetivo & Não efetivo \\
\hline $\begin{array}{l}\text { Programa nutricional } \\
\text { e AF }\end{array}$ & $\begin{array}{l}\text { Bacardí-Gascon } \\
\text { et al. }{ }^{34}\end{array}$ & --- & Muros et al. ${ }^{30}$ & \begin{tabular}{|l} 
Rush et al. ${ }^{27}$ \\
Hrafnkelsson et al. ${ }^{31}$
\end{tabular} \\
\hline $\begin{array}{l}\text { Programa nutricional, } \\
\text { AF e sessões educativas } \\
\text { em sala de aula }\end{array}$ & $\begin{array}{l}\text { Colín-Ramírez } \\
\text { et al. }{ }^{33}\end{array}$ & Morgan et al..$^{35}$ & $\begin{array}{l}\text { Militão et al. }{ }^{29} \\
\text { Angelopoulo et al. }{ }^{20}\end{array}$ & $\begin{array}{l}\text { Choudhry et al. } .^{25} \\
\text { Plachta-Danielzik } \\
\text { et al. }{ }^{24}\end{array}$ \\
\hline $\begin{array}{l}\text { Programa nutricional } \\
\text { e sessões educativas em } \\
\text { sala de aula }\end{array}$ & $\begin{array}{l}\text { Cotter et al. }{ }^{18} \\
\text { He et al. }{ }^{32}\end{array}$ & --- & --- & He et al..$^{32}$ \\
\hline $\begin{array}{l}\text { Programa nutricional, } \\
\text { AF e atividades de } \\
\text { jardinagem }\end{array}$ & --- & --- & Hollar et al. ${ }^{23}$ & Hollar et al. ${ }^{22}$ \\
\hline $\begin{array}{l}\text { Programa nutricional, } \\
\text { AF e apoio psicológico }\end{array}$ & --- & --- & $\begin{array}{l}\text { Reinehr et al. }{ }^{21} \\
\text { Schaefer et al. }{ }^{26} \\
\text { Vásquez et al. }{ }^{28}\end{array}$ & --- \\
\hline
\end{tabular}

*PA: pressão arterial, AF: atividade física.

Entre os trabalhos que apresentaram efetividade em seus resultados, notou-se que a maioria realizou a intervenção em tempo igual ou superior a seis meses ${ }^{18,20,21,23,26,30,33,34} \mathrm{e}$, quase a metade dos trabalhos que não apresentaram resultados efetivos apresentaram período de tempo inferior a seis meses ${ }^{25,32,35}$.

\section{Discussão}

A maioria dos estudos analisados relacionou duas ou mais práticas educativas, principalmente em relação às atividades de educação nutricional e atividade física. Entretanto, notaram-se algumas diversidades de resultados, como verificado por Vásquez et al. ${ }^{28}$. Nesta pesquisa, os autores observaram um aumento $(\mathrm{p}=0,003)$ da pressão arterial no grupo que recebeu apenas informação e motivação nutricional e psicológica. Contrariamente, Reinehr et al. $^{21}$ relataram redução ( $p$ $<0,001$ ) da PAS e PAD após treinamento com atividade física, educação nutricional e aconselhamento comportamental. Assim, apesar dos estudos apresentarem objetivos similares (avaliar o efeito de programas de intervenção sobre aspectos relacionados à saúde do público infanto-juvenil), houve algumas diferenciações entre as questões abordadas na intervenção, como a presença do exercício físico ${ }^{21}$. Diante disso, os próprios autores ${ }^{28}$ esclarecem a importância e a relação entre os exercícios de força muscular propostos, com a diminuição subsequente de fatores de risco como, pressão arterial, peso corpóreo, gordura corporal e circunferência abdominal no grupo que recebeu a intervenção completa e de maior extensão (informação e motivação nutricional e psicológica juntamente com sessões com exercício físico) nos três meses seguintes de intervenção, comparado àquele que recebeu somente informação e motivação nutricional e psicológica nos primeiros três meses.

O intervalo de tempo das ações pode ser outro fator que interfere na eficácia dos resultados. Ao se analisar o período de intervenção, observou-se que quatro pesquisas tiveram duração inferior a seis meses, obtendo pouca efetividade na modificação da pressão arterial ou no consumo de alimentos com alto teor de sódio ${ }^{25,32,35}$. Neste aspecto, a eficácia de ações com duração prolongada já é reconhecida na literatura ${ }^{36,37}$. Segundo Wendy et al. ${ }^{38}$, as práticas de curta duração são menos adequadas devido à fatores não relacionados ao ambiente escolar, como a mídia, videogame e redes de fast foods, os quais também exercem forte influência sobre os hábitos infantis. Além disso, Fernandes et al. ${ }^{39}$ explicam que o processo de modificação dos hábitos alimentares nas crianças necessita de um longo período de tempo para refletir-se em alguma alteração. Dessa forma, nota-se a grande influência que o tempo de duração das práticas interdisciplinares pode exercer sobre os componentes relacionados à saúde dos escolares. Apesar destas constatações, Souza et al. ${ }^{40}$ demonstraram que a efetividade das intervenções foi independente do tempo de du- 
ração dos programas. O mesmo pôde ser constatado em estudo apresentado nesta revisão ${ }^{24}$.

Ações aplicadas em escolas, com foco na saúde da criança, têm buscado uma atenção integral, visando atingir vários fatores relacionados ao adequado estilo de vida. Desta forma, trabalhos que inter-relacionam diferentes áreas, como educação física e nutrição, junto às disciplinas escolares ${ }^{41}$, bem como os diversos sujeitos que exercem influência sobre a saúde da criança, como professores e familiares $^{34,42}$, têm sido cada vez mais praticados. Além disso, especialistas recomendam a combinação do incentivo a prática de exercícios físicos, nutrição, educação e terapia comportamental nas intervenções ${ }^{43-45}$. A importância de unir estas atividades em um programa interdisciplinar ficou evidente na pesquisa de Muros et al. ${ }^{30}$. As ações eram realizadas com crianças obesas, por 6 meses. Nos 3 meses iniciais de intervenção, o grupo 1, que recebia apenas educação nutricional e psicológica, apresentou aumento da pressão arterial $(\mathrm{p}=0,02)$, comparado ao grupo 2 no qual eram aplicados, também, exercícios físicos, aliados às outras duas atividades. Nos 3 meses seguintes, o grupo 1 começou a receber orientações de exercícios físicos, porém sem as ações de educação nutricional e psicológica. Esse fato reduziu $(\mathrm{p}=0,01)$ a pressão arterial, em comparação ao grupo 2, que apresentou elevação da pressão ( $\mathrm{p}=0,003)$, pois passou a não receber nenhum tipo de intervenção. Nesse contexto, as ações interdisciplinares mostram-se altamente eficazes na promoção da saúde, uma vez que repercutem diretamente sobre os conhecimentos, atitudes e opiniões das crianças ${ }^{33,35}$. Da mesma forma, influenciam na menor ingestão de alimentos contendo altos teores de gordura, sal e calorias $^{20,29,33}$, aumento do nível de atividade físi$\mathrm{ca}^{29,33} \mathrm{e}$, consequente, redução do peso corporal ${ }^{46}$.

Considera-se que mudanças no comportamento dos pais podem incentivar as crianças a obterem hábitos de vida saudáveis ao longo da vida $^{34}$. Ao mesmo tempo, a inclusão de atividades interativas e lúdicas pelos professores promovem a adoção de um estilo de vida saudável pelos alunos ${ }^{47}$. Nesse aspecto, a inclusão de outros indivíduos nas práticas interdisciplinares, foram considerados em grande parte dos estudos avaliados ${ }^{20,22,23,24,27,31,32,33,34,35}$. Apesar disso, foi possível constatar maior efetividade em alguns programas que abrangeram somente a família ${ }^{21,26,28,30} \mathrm{ou}$ professores $^{18,29}$, quando comparados àqueles que envolveram conjuntamente os pais e professores $^{24,25,31,32}$ e a comunidade local ${ }^{27}$. Salienta-se que apesar do envolvimento com outros indivíduos, a participação dos pais não foi regular como se almejava, sendo que o maior contato foi por meio do envio de materiais informativos ${ }^{25}$. Além disso, fatores como o não monitoramento do comportamento das crianças fora do ambiente escolar ${ }^{27}$, alta taxa de abandono durante a intervenção ${ }^{32}$, o autorrelato dos estudantes sobre seus comportamentos $^{25}$ (o que não garante a veracidade das respostas), e o não acompanhamento dos mesmos após o período da intervenção ${ }^{24}$, não permitem uma avaliação real tanto das mudanças de comportamento alimentar e de atividade física, bem como mudanças efetivas na PA após o período estipulado. Assim, compreendem-se estes fatores como possíveis explicações para a efetividade de alguns trabalhos em detrimento de outros.

Ao se analisar a efetividade dos artigos analisados, observou-se que a maioria deles (7) $20,21,23,26,28,29,32,33$ empregou mais de duas práticas interdisciplinares conjuntas em seus programas, atingindo melhores resultados, isso quando comparados àqueles $(3)^{18,30,31,34}$ que utilizaram somente duas ações. Porém, a realização das estratégias interdisciplinares, normalmente, ocorria de forma simultânea, o que dificultava uma análise isolada sobre o impacto individual no estilo de vida da criança ${ }^{21}$. Assim, não se pode relacionar totalmente a presença de um número maior de intervenções com resultados mais positivos. Esse fato foi observado na pesquisa de Morgan et al. ${ }^{35}$, que avaliaram ferramentas educacionais e motivacionais (avatares), por meio de computadores, junto à educação nutricional e atividade física, sendo que não foi possível reduzir o consumo de alimentos com elevado teor de sódio. Porém, Cotter et al. ${ }^{18}$, que utilizaram apenas ações de educação nutricional, conseguiram atingir todos objetivos propostos. Resultados como estes também dificultam a comparação entre os estudos, prejudicando igualmente a concordância com a literatura ${ }^{34,47}$. Apesar desta constatação, considerara-se a hipertensão arterial como um problema multifatorial ${ }^{48}$, tornando-se necessária a união de diferentes abordagens que incidam sobre o estilo de vida da criança, bem como sobre os fatores dietéticos ${ }^{29}$, sedentarismo ${ }^{33}$, apoio psicológico ${ }^{28} \mathrm{e}$ aconselhamento comportamental ${ }^{21,26}$.

Em relação à metodologia utilizada, foi observado que alguns programas de intervenção se basearam em outros pré-existentes ${ }^{21,26}$. Assim, destaca-se a proposta "Obeldicks light" de Reinehr et $\mathrm{al}^{21}$ e Schaefer et al. ${ }^{26}$, os quais fundamentaram-se na intervenção "Obeldicks" para crianças e adolescentes obesos ${ }^{49}$. O programa se mostrou efetivo, estando totalmente relacionado a uma 
proposta interdisciplinar no ambiente escolar. Baseado em práticas que intervêm diretamente no estilo de vida de crianças com excesso de peso e seus pais, por meio de ações nas áreas de educação nutricional, atividade física e aconselhamento comportamental, incluindo atendimento psicológico individual. As ações eram desenvolvidas por uma equipe interdisciplinar, que buscava promover diferentes atividades, com foco a obter resultados em diversos parâmetros relacionados à saúde metabólica da criança, como peso, composição de gordura corporal, circunferência da cintura e pressão arterial sistólica e diastólica. Já, o método "Obeldicks light" pretende trabalhar o comportamento geral do indivíduo, assemelhando-se a pesquisa de Choudhry et al. ${ }^{25}$, que aplicaram o programa Power-Up, o qual se baseia em fatores sociais, ambientais e da comunidade que podem impactar o comportamento do indivíduo, e também à pesquisa de Angelopoulos et al. ${ }^{20}$, que tinha o objetivo de avaliar e modificar hábitos relacionados à autoestima, imagem corporal, nutrição, atividade física e voltados à questões ambientais das crianças, através do estudo da Teoria do Comportamento Planejado. Este conceito defende que a intenção de um indivíduo de exercer determinado comportamento pode estar baseada em um conjunto de construtos, como: a) pressão social dos pais, amigos, cultura e instituições; b) crença em fatores que podem facilitar ou impedir um comportamento e; c) interesse sobre as consequências geradas após o comportamento $\operatorname{adotado}^{50}$. Portanto, fazem uso de uma visão interdisciplinar para compreender as diferentes influências que cercam o comportamento de estilo de vida adotado pelo público infantil. Isso pode ser justificado pela presença dos pais e professores nas atividades e, pela valorização de espaços como a escola e parques de diversão como ambientes importantes na formação dos comportamentos em saúde da criança ${ }^{20}$.

Alguns estudos analisados adotaram em seu método intervencionista estreita colaboração com outros serviços, como, por exemplo, a administração escolar, as cantinas, a Extensão Universitária, por meio da presença de professores de Nutrição, o Serviço de Alimentação e Nutrição do Departamento de Agricultura dos Estados Unidos (USDA), Revistas (ex. Organic Gardening) e, Conselhos de Saúde (ex. Waikato District Health Board), dentre outros ${ }^{22,23,31,36}$. Esta relação entre as áreas tinha o objetivo de contribuir na aplicação dos programas e obter maior êxito nas atividades, o que foi confirmado pelos resultados positivos obtidos nas pesquisas. Assim, este tipo de metodologia também se mostrou satisfatória e válida para a obtenção dos resultados esperados.

Em outros programas de intervenção foram utilizados currículos estruturados, como forma de empregar as atividades educacionais ${ }^{25,27,34,35}$. No trabalho de Rush et al. ${ }^{27}$ e Choudhry et al. ${ }^{25}$, os currículos eram utilizados para fornecer informações sobre orientações alimentares saudáveis e ideias de jogos, atividades e dinâmicas, respectivamente, sendo aplicados nas escolas com foco na alimentação e atividade física. Os resultados obtidos com estas duas técnicas foram positivos quando relacionados ao excesso de peso, uma vez que se obteve a diminuição de gordura corporal ${ }^{27}$ e $\mathrm{IMC}^{25}$, mas não houve uma redução significativa da pressão arterial das crianças envolvidas nos grupos de intervenção. Já, Bacardí-Gascon et al. ${ }^{34}$ utilizaram como estratégia de intervenção o Modelo Ecológico de Bronfenbrenner, para organização do currículo. Este instrumento permite compreender o desenvolvimento humano aliado ao ambiente físico, social e cultural, onde a criança está inserida.

Partindo desta visão ampla, procura-se conceber, também, qual o lugar assumido pela escola no desenvolvimento infantil ${ }^{51}$. Estudo de Militão et al. ${ }^{29}$, desenvolvido em Brasília, ocorreu em ambiente escolar e, atribuiu isto ao fato de nenhuma criança desistir da intervenção proposta durante a pesquisa. Assim, o ambiente escolar no Brasil deve ser reconhecido como propício para o desenvolvimento de práticas em saúde, uma vez que as crianças permanecem grande parte do seu tempo na escola ${ }^{52}$. Além disso, por ser um local de intensa interação social, que permite tanto a oferta de uma alimentação balanceada, a qual nem sempre a criança tem acesso em sua casa, quanto um espaço adequado para a realização de diferentes tipos de atividades, que muitas vezes não podem ser praticadas fora da escola devido à crescente violência urbana, torna-se essencial para o estímulo a uma vida mais saudável de uma forma mais abrangente $\mathrm{e}^{53}$. Têm-se, portanto, um ambiente central para a reestruturação e aquisição de novos conhecimentos, seja por meio de um diálogo entre aluno e professor ${ }^{54}$, ou pela relação que se pode estabelecer entre as crianças e suas famílias dentro do espectro social escolar ${ }^{55}$.

Diante desta revisão, alguns fatores devem ser considerados ao se avaliar um programa de intervenção desenvolvido no ambiente escolar, visto a complexidade do contexto educativo ${ }^{56}$. Cita-se, inicialmente, o foco da intervenção, ou seja, a abordagem das ações deve ocorrer de maneira interdisciplinar. Isto se deve tanto à necessidade de 
interação entre os profissionais ${ }^{56}$, como também à um comprometimento ambiental extenso na vida das crianças, incluindo seus lares, cantinas escolares e, até mesmo, atividades extra classe ${ }^{35}$. Também, destaca-se o período que ocorre durante a intervenção. Neste aspecto, embora não se tenha estipulado um tempo ideal até o momento, a literatura recomenda programas com duração prolongada, para que ocorram mudanças sustentáveis no estilo de vida do público infantil e adolescente $^{57}$. Entretanto, pode haver influência de outros aspectos, como o alto custo das intervenções, fato que pode limitar o tempo de duração, o número amostral, o local e a qualidade metodológica da intervençãa ${ }^{58}$.

\section{Conclusão}

A revisão sistemática identificou uma maioria de trabalhos nos quais as práticas disciplinares na escola se mostraram eficazes para a melhoria dos níveis de pressão arterial e para a redução do consumo de alimentos com alto teor em sódio e/ ou sal por crianças. Assim, a união de orientações nutricionais com atividade física mostrou-se adequada para atingir os resultados. Porém, estas ações são mais efetivas, na maioria dos estudos avaliados, quando realizadas por um período maior de tempo.

Os trabalhos demonstraram envolver nas atividades propostas e pessoas pertencentes ao entorno escolar, uma vez que exercem forte influência sobre os hábitos de vida das crianças. A participação efetiva, principalmente de pais e professores, na maioria dos trabalhos realça a importância de práticas continuadas, adotadas no ambiente familiar, ampliando o aprendizado.

É necessária a realização de mais estudos que realizem uma comparação entre diferentes intervenções e sua duração, para a prevenção da hipertensão arterial em anos posteriores à infância, permitindo que estas ações possam ser adotadas rotineiramente nos currículos escolares.

\section{Colaboradores}

L Bernardi, MC França, AM Xavier e D Novello participaram da concepção e das revisões que resultaram neste artigo. 


\section{Referências}

1. Martins TL, Atallah AN, Silva EMK. Blood pressure control in hypertensive patients within Family Health Program versus at Primary Healthcare Units: analytical cross-sectional study. Sao Paulo Med J 2012; 130(3):1516-3180.

2. World Health Organization. Health statistics and information systems [Internet]. Geneva: World Health Organization; 2014 [cited 2014 Dec 15]. Available from: http://www.who.int/healthinfo/global_burden_disease/estimates/en/index1.html

3. Brasil. Ministério da Saúde (MS). Vigitel 2013: vigilância de fatores de risco e proteção para doenças crônicas por inquérito telefônico. Brasília: MS; 2013.

4. Lubrano R, Travasso E, Raggi C, Guido G, Masciangelo R, Elli M. Blood pressure load, proteinuria and renal function in pre-hypertensive children. Pediatr Nephrol 2009; 24(4):823-831.

5. Leshem M. Biobehavior of the human love of salt. Neurosci Biobehav Rev 2009; 33(1):1-17.

6. Yang Q, Zhang Z, Kuklina EV, Fang J, Ayala C, Hong Y, Loustalot F, Dai S, Gunn JP, Tian N, Cogswell ME, Merritt R. Sodium Intake and Blood Pressure Among US Children and Adolescents. Pediatrics 2012; 130(4):611-619.

7. Kelishadi R, Gheisari A, Zare N, Farajian S, Shariatinejad K. Salt intake and the association with blood pressure in young Iranian children: first report from the middle East and north Africa. Int J Prev Med 2013; 4(4):475-483.

8. Shi L, Krupp D, Remer T. Salt, fruit and vegetable consumption and blood pressure development: a longitudinal investigation in healthy children. Br J Nutr 2014; 111(4):662-671.

9. Norman MK, Victor RG. Clinical Hypertension. $10^{\text {th }} \mathrm{ed}$. Philadelphia; 2010.

10. Nguyen T, Lau DC. The Obesity Epidemic and Its Impact on Hypertension. Can J Cardiol 2012; 28(3):326-333.

11. Haddy FJ. Role of dietary salt in hypertension. J Am Coll Nutr Life Sci 2006; 79(17):1585-1592.

12. Costa FP, Machado SH. O consumo de sal e alimentos ricos em sódio pode influenciar na pressão arterial das crianças? Cien Saude Colet 2010; 15(1):1383-1389.

13. Grimes CA, Campbell KJ, Riddell LJ, Nowson CA. Sources of sodium in Australian children's diets and the effect of the application of sodium targets to food products to reduce sodium intake. Br J Nutr 2011; 105(3):468-477.

14. Toschke AM, Kohl L, Mansmann U, von Kries R. Meta-analysis of blood pressure tracking from childhood to adulthood and implications for the design of intervention trial. Acta Paediatr 2010; 99(1):24-29.

15. Drewnowski A, Rehm CD. Sodium Intakes of US Children and Adults from Foods and Beverages by Location of Origin and by Specific Food Source. Nutrients 2013; 5(6):1840-1855.

16. He FJ, Wu Y, Ma J, Feng X, Wang H, Zhang J, Lin CP, Yuan J, Ma Y, Yang Y, Yan LL, Jan S, Nowson C, Macgregor GA. A school-based education programme to reduce salt intake in children and their families (School-EduSalt): protocol of a cluster randomised controlled trial. BMJ 2013; 3(7):1-8.

17. Piziak V. A pilot study of a pictorial bilingual nutrition education game to improve the consumption of healthful foods in a head start population. Int J Environ Res Public Health 2012; 9(4):1319-1325.
18. Cotter J, Cotter MJ, Oliveira P, Cunha P, Polónia J. Salt intake in children 10-12 years old and its modification by active working practices in a school garden. J Hypertens 2013; 31(10):1966-1971.

19. Association of University Centers on Disabilities (AUCD). Research, Education, Service. Interdisciplinary practice. [internet]. 2007 [ acessado 2015 Jan 19] . Disponível em: http://www.aucd.org/template/index. $\mathrm{cfm}$

20. Angelopoulos PD, Milionis HJ, Grammatikaki E, Moschonis G, Manios Y. Changes in BMI and blood pressure after a school based intervention: the CHILDREN study. Eur J Public Health 2009; 19(3):319-325.

21. Reinehr T, Schaefer A, Winkel K, Finne E, Toschke AM, Kolip P. An effective lifestyle intervention in overweight children: findings from a randomized controlled trial on "Obeldicks light". Clin Nutr 2010; 29(3):331-336

22. Hollar D, Messiah SE, Lopez-Mitnik G, Hollar TL, Almon M, Agatston AS. Healthier Options for Public Schoolchildren Program Improves Weight and Blood Pressure in 6- to 13-Year-Olds. J Am Diet Assoc 2010; 110(2):261-267.

23. Hollar D, Lombardo M, Lopez-Mitnik G, Hollar TL, Almon M, Agatston AS, Messiah SE. Effective Multi-level, Multi-sector, School-based Obesity Prevention Programming Improves Weight, Blood Pressure, and Academic Performance, Especially among Low-Income, Minority Children. J Health Care Poor Underserved 2010; 21(Supl. 2):93-108.

24. Plachta-Danielzik S, Landsberg B, Lange D, Seiberl J, Muller MJ. Eight-Year Follow-Up of School-Based Intervention on Childhood Overweight - the Kiel Obesity Prevention Study. Europ J Obes Facts 2011; 4(1):3543.

25. Choudhry S, McClinton-Powell L, Solomon M, Davis D, Lipton R, Darukhanavala A, Burnet DL, Steenes A, Selvaraj K, Gielissen K, Love L, Salahuddin R, Embil FK, Dezheng Huo, Chin MH, Quinn MT, Brunet D. Power-Up: A Collaborative After-School Program to Prevent Obesity in African American Children. Prog Community Health Partnersh 2011; 5(4):363-373.

26. Schaefer A, Winkel K, Finne E, Kolip P, Reinehr T. An effective lifestyle intervention in overweight children: one-year follow-up after the randomized controlled trial on "Obeldicks light". Clin Nutr 2011; 30(5):629633.

27. Rush E, Reed P, McLennan S, Coppinger T, Simmons D, Graham D. A school-based obesity control programme: Project Energize. Two-year outcomes. $\mathrm{Br} \mathrm{J}$ Nutr 2012; 107(4):581-587.

28. Vásquez F, Díaz E, Lera L, Meza J, Salas I, Rojas P, Atalah E, Burrows R. Impacto del ejercicio de fuerza muscular en la prevención secundaria de la obesidad infantil; intervención al interior del sistema escolar. Nutr Hosp 2013; 28(2):347-356.

29. Militão AG, Karnikowski MGO, Da Silva FR, Militão ESG, Pereira RMS, Campbell CSG. Effects of a recreational physical activity and healthy habits orientation program, using an illustrated diary, on the cardiovascular risk profile of overweight and obese schoolchildren: a pilot study in a public school in Brasilia, Federal District, Brazil. Diabetes Metab Syndr Obes 2013; 6(1):445-451. 
30. Muros JJ, Zabala M, Oliveiras-López MJ, López-Garcia de La Serrana H. Effect of physical activity, nutritional education, and consumption of EVOO on lipid, physiological, and anthropometric profiles in a pediatric population. Pediatr Exerc Sci 2013; 25(2):248-261.

31. Hrafnkelsson H, Magnusson KT, Thorsdottir I, Johannsson E, Sigurdsson EL. Result of school-based intervention on cardiovascular risk factors. Scand J Prim Health Care 2014; 32(4):149-155.

32. He FJ, Wu Y, Feng X, Ma J, Ma Y, Wang H, Zhang J, Yuan J, Lin C, Nowson C, MacGregor GA. School based education programme to reduce salt intake in children and their families (School-EduSalt): cluster randomized controlled Trial. BMJ 2015; 315(1):770.

33. Colín-Ramírez E, Castillo Martínez L, Orea Tejeda A, Vergara A, Villa AR. Efecto de una intervención escolar basada en actividad física y dieta para la prevención de factores de riesgo cardiovascular (RESCATE). Rev Esp Nutr Comunitaria 2009; 15(2):71-80.

34. Bacardí-Gascon, M, Pérez-Morales ME, Jiménez-Cruz A. A six month randomized school intervention and an 18-month follow-up intervention to prevent childhood obesity in Mexican. Nutr Hosp 2012; 27(3):755-762.

35. Morgan AZ, Ulrich P, Simmons KP, Gropper SS, Connell LJ, Daniels MK, Latham E, Keiley MK. Effectiveness of a multi-faceted, school-based health intervention program with 4th graders in Alabama. CYSR 2014; 37(1):46-54.

36. Meyer AA, Kundt G, Lenschow U, Schuff-Werner P, Kienast W. Improvement of early vascular changes and cardiovascular risk factors in obese children after a six-month exercise program. J Am Coll Cardiol 2006; 48(9):1865-1870.

37. Wunsch R, de Sousa G, Toschke AM, Reinehr T. Intima-media thickness in obese children before and after weight loss. Pediatrics 2006; 118(6):2334-2340.

38. Wendy TRW, Jones LE, Williams SM, Goulding A. Evaluation of waist circunference, waist-to-hip ratio and the conicity index as screening tolls for high trunk fat mass as measured by dual energy $\mathrm{X}$ ray absorptiometry in children aged 3-19 years. Am J Clin Nutr 2006; 72:490-495.

39. Fernandes PS, Bernardo CO, Campos RMMB, Vasconcelos FAG. Avaliação do efeito da educação nutricional na prevalência de sobrepeso/obesidade e no consumo alimentar de escolares do ensino fundamental. J Pediatr 2009; 85(4):315-321.

40. Souza EA, Filho VCB, Nogueira JAD, Júnior MRA. Atividade física e alimentação saudável em escolares brasileiros: revisão de programas de intervenção. Cad Saude Publica 2011; 27(8):1459-1471.

41. Kipping RR, Howe LD, Jago R, Campbell R, Wells S, Chittleborough CR, Mytton J, Noble SM, Peters TJ, Lawlor DA. Effect of intervention aimed at increasing physical activity, reducing sedentary behaviour, and increasing fruit and vegetable consumption in children: Active for Life Year 5 (AFLY5) school based cluster randomised controlled trial. BMJ 2014; 348:3256.

42. Doyle-Baker PK, Venner AA, Lyon ME, Fung Tn. Impact of a combined diet and progressive exercise intervention for overweight and obese children: the B.E. H.I.P. study. Appl Physiol Nutr Metab 2011; 36(4):515525.

43. Braet C, Winckel MV, Leeuwen KV. Follow-up results of different treatment programs for obese children. Acto Pediatr 1997; 86(4):397-402.
44. Suskind RM, Blecker U, Udall J, von Almen TK, Schumacher HD, Carlisle L, Sothern MS. Recent advances in the treatment of childhood obesity. Pediatr Diabetes 2000; 1(1):23-33.

45. Reinehr T, Kersting M, Alex U, Andler W. Long-term follow-up of overweight children: after training, after a single consultation session, and without treatment. $J$ Pediatr Gastroenterol Nutr 2003; 37(1):72-74.

46. Farris JW, Taylor L, Williamson M, Robinson C. A 12-week interdisciplinary intervention program for children who are obese. Cardiopulm Phys Ther J 2011; 22(4):12-20

47. Van Cauwenberghe E, Maes L, Spittaels H, van Lenthe FJ, Brug J, Oppert JM, De Bourdeaudhuij I. Effectiveness of school-based interventions in Europe to promote healthy nutrition in children and adolescents: systematic review of published and "grey" literature. $\mathrm{Br}$ J Nutr 2010; 103(6):781-797.

48. Sociedade Brasileira de Hipertensão (SBH), Sociedade Brasileira de Cardiologia (SBC), Sociedade Brasileira de Nefrologia (SBN). III Consenso Brasileiro de Hipertensão Arterial. Arq Bras Endocrinol Metab 1999; 43(4):257-286.

49. Reinehr T, Kersting M, Wollenhaupt A, Alexy U, Kling B, Strobele K, Andler W. Evaluation of the training program "OBELDICKS" for obese children and adolescents. Klin Padiatr 2005; 217(1):1-8.

50. Souza GFM, Borges FRF, Lopes ALM, Muniz RA. Propagação de mensagens na internet: Teoria do Comportamento Planejado. Pretexto 2012; 13(3):11-27.

51. Mendes LSA. A escola enquanto contexto de desenvolvimento: um estudo ecológico em uma comunidade ribeirinha na ilha do Marajó [tese]. Belém: Universidade Federal do Pará; 2008.

52. Cruz OLM, Costa SS. Disacusias Neurossensoriais Induzidas por Ruído. In: Cruz OLM, Costa SS, Oliveira JAA. Otorrinolaringologia: Princípios e prática. Porto Alegre: Artmed; 1994.

53. Enes CC, Slater B. Obesity in adolescence and its main determinants. Rev Bras Epidemiol 2010; 13(1):163-171.

54. De Azevedo MAR, De Andrade MFR. O conhecimento em sala de aula: a organização do ensino numa perspectiva interdisciplinar. Educ Rev 2007; 30(1):235-250.

55. Lloyd JJ, Logan S, Greaves CJ, Wyatt KM. Evidence, theory and context-using intervention mapping to develop a school-based intervention to prevent obesity in children. Int J Behav Nutr Phys Act 2011; 8(73)1-15.

56. Carvalho RGG. A dimensão relacional da intervenção dos serviços de psicologia nas escolas. Psicol Reflex Crit 2008; 21(1):119-124.

57. Kamath CC, Vickers KS, Ehrlich A, McGovern L, Johnson, J, Singhal V, Paulo R, Hettinger A, Erwin PJ, Montori VM. Behavioral interventions to prevent childhood obesity: a systematic review and metaanalyses of randomized trials. J Clin Endocrinol Metab 2008; 12(1):4606-4615

58. Jones SE, Brener ND, McManus T. Prevalence of school policies, programs, and facilities that promote a healthy physical school environment. Am J Public Health 2003; 93(9):1570-1575.

Artigo apresentado em 14/05/2015

Aprovado em 11/05/2016

Versão final apresentada em 13/05/2016 